\title{
Outcome of patients with chronic migraine with medication overuse and depression after duloxetine: influence of coexisting obsessive compulsive disorder
}

\author{
Marcella Curone • V. Tullo • M. Savino • \\ A. Proietti-Cecchini - G. Bussone - D. D’Amico
}

(C) Springer-Verlag Italia 2013

\begin{abstract}
Patients with chronic migraine (CM) and medication overuse (MO) have high frequency of psychiatric comorbidity. Aims of this open label, prospective, independent study were: to evaluate the efficacy of duloxetine in a sample of patients with MO due to CM and with concomitant depression; to investigate, if the presence of OCD influences the outcome in this subgroup of patients. A total of 50 consecutive patients $(40 \mathrm{~F}, 10 \mathrm{M}$, aged 20-65 years, mean 39.4 years) from those attending our Headache Center to undergo an inpatient withdrawal programme followed by anti-migraine prophylaxis was enrolled. After a 1-month baseline period, all patients were prescribed duloxetine $30 \mathrm{mg}$ in the morning for the first week, and $60 \mathrm{mg}$ for the following 12 weeks. They filled a daily headache diary during the whole study period. They also completed Hamilton depression rating scale (HDRS) and migraine disability assessment scale (MIDAS) at baseline and at the 12-week follow-up. The primary outcome measure was the percentage of responders, i.e. of patients with a reduction $\geq 50 \%$ in headache frequency as well as in symptomatic drug consumption. Comparison between patients with and without OCD was performed. Our results showed a rather high responder rate in the total sample (64\%), while none of the patients with OCD fell among responders. MIDAS and HDRS scores had a more
\end{abstract}

M. Curone $(\varangle)$. V. Tullo · A. Proietti-Cecchini .

G. Bussone · D. D'Amico

Department of Clinical Neuroscience, Headache Unit,

C. Besta Neurological Institute and Fundation,

Via Celoria 11, 20133 Milan, Italy

e-mail: marcella.msc.doc@gmail.com

M. Savino

Visconti di Modrone Psychiatric and Mental Health Center,

Via Visconti di Modrone 7, Milan, Italy evident decrease in patients without OSD. These findings suggest that duloxetine may be effective in patients with MO due to CM and with comorbid depression. They also confirm the importance of a systematic assessment of the psychopathological profile in these patients, and indicate that clinicians should be aware of the relevant prognostic role of OCD in favoring a poor outcome and persistent disability in headache patients with MO.

Keywords Chronic migraine (CM) .

Medication overuse (MO) - Duloxetine · Depression . Obsessive compulsive disorder (OCD) · Outcome

\section{Introduction}

Patients with chronic migraine (CM) and medication overuse (MO) have high frequency of psychiatric comorbidity, the presence of which can influence prognosis and response to treatment [1-3]. Antidepressants are often used to treat this condition in clinical practice $[4,5]$. Obsessive compulsive disorder (OCD) is mentioned among comorbid conditions in patients with $\mathrm{CM}$ and MO [6, 7], although data are lacking as how to manage this aspect in the treatment of headache patients.

Both depressive and anxiety disorders may play an important role in the peripheral and central mechanisms of pain sensitization which may contribute to the evolution of episodic migraine to $\mathrm{CM}$, as well in promoting MO [8]. OCD is classified under the group of anxiety disorders and it is characterized by recurrent obsessive thoughts and repetitive behaviors adopted to relieve the anxiety caused by the obsessive thoughts [9]. It has been suggested that its presence in $\mathrm{CM}$ and $\mathrm{MO}$ is likely to complicate the clinical course and may cause a poor response to treatment [10]. 
The aims of this study were: to evaluate the efficacy of duloxetine in a sample of patients with MO due to CM and with concomitant depression and to investigate if the presence of OCD influences the outcome in this subgroup of patients.

\section{Patients and methods}

This was an open label, prospective, independent study.

A consecutive sample of patients from those attending our Headache Center to undergo an inpatient withdrawal programme to stop MO was enrolled. Inclusion criteria were: diagnosis of $\mathrm{MO}$ according to ICDH-2 revised criteria [11]; history of CM, according to ICDH-2 revised criteria [11]; and presence of major depression. All patients were asked to complete Hamilton depression rating scale (HDRS) and migraine disability assessment scale (MIDAS) before discharge. All patients were evaluated by a senior psychiatrist to confirm a diagnosis of major depression as well as the presence of OCD. After discharge, pharmacological prevention was started, choosing a compound from those included in the current guidelines for migraine prophylaxis, but avoiding antidepressant compounds [12]. Patients had a baseline-1 month period after discharge, during which they filled an daily headache diary with recording of presence and severity of headache and symptomatic medication consumption. At the end of baseline-period all patients were prescribed duloxetine $30 \mathrm{mg}$ in the morning for the first week, and $60 \mathrm{mg}$ for the following 12 weeks. They were asked to fill the daily headache diary during the whole study period. Patients were assessed after 4, 8 and 12 weeks with scheduled follow-up visits. At the end of the treatment period data from the structured diaries were collected and both HDRS and MIDAS were performed in each patient.

The primary outcome measure was the responder rate, i.e. the proportion of patients with a reduction $\geq 50 \%$ in headache frequency as well as in symptomatic drug consumption. Other outcome measures used to assess the outcome were: headache intensity (rated with VAS); HDRS score; MIDAS score. Comparisons between all the above mentioned data as found at baseline and at the 12-week follow-up were made, in terms of percentages of responders for frequency of headache and symptomatic drug consumption, and in terms of numerical findings (mean value and SD) for the remaining outcome measures. Comparisons of the percentage of responders was also made between patients with and without OCD.

\section{Results}

A total of 50 patients (40 women and 10 men, aged 20-65 years, mean 39,4 years) entered the study. Presence of major depression was confirmed in all subjects, and in 14 patients a diagnosis of OCD was also made. HDRS score was between 20 and 35, and MIDAS score was higher than 21 (grade IV) in all patients. All patients completed the study and filled the structured diaries in each part. Duloxetine was well tolerated, with no major adverse event reported by any patient.

The responder rate was $64 \%$ (32/50 patients). As shown in Table 1 , most of the patients who were responders had an improvement higher than $50 \%$. A reduction of at least $50 \%$ in headache intensity was found in the same number of patients after the treatment period. In fact, all the studied outcome measures showed a marked improvement in these 32 patients after duloxetine treatment as the number of headache days per month changed from 20.6, SD 1.8-11.0, SD 1.3; the number of analgesic per month decreased from 20.0, SD 1.8-10.5, SD 1.3; HDRS and MIDAS scores were markedly ( $\geq 50 \%$ ) reduced at the end of the study period: (HDRS score decreased from 26.3, SD 2.0-14.8, SD 1.1, and MIDAS score from 85.66, SD 21.4-6.7, SD 2,05).

None of the patients with OCD fell among responders. We note that HDRS score showed a lower improvement in OCD patients than in those without OCD. The same trend was observed for MIDAS score. Futhermore, we observed that patients with OCD showed a more complex headache report with reiteration of the obsessive thoughts about their pain. They also manifested an overrating expectation on new-starting treatment, and exhibited at the same time doubts about treatment outcome during the psychiatric interview, often reporting these feelings in their daily diaries.

\section{Discussion}

Duloxetine is a selective serotonin and norepinephrine reuptake inhibitor with proven efficacy in major depression [13, 14].

Our findings showed that this compound may be effective in reducing headache frequency and in limiting $\mathrm{MO}$ in patients with $\mathrm{CM}$ and coexisting depression, when prescribed after inpatient detoxification programme and the start of an anti-migraine prophylaxis.

The improvement was evident not only in depression scores, but also in all headache parameters, with a high rate of responders as far as frequency and symptomatic consumption reduction, and improvements in the other studied parameters, i.e. pain intensity and disability in daily activities.

On the other hand, a poor outcome was observed in those patients with coexistent OCD, with no patient achieving the $50 \%$ reduction in either headache frequency or consumption of symptomatic medications. 
Table 1 Number of responders and responder rates after treatment

\begin{tabular}{lccc}
\hline Responders & Total & Patients without OCD & Patients with OCD \\
\hline Reduction in monthly headache frequency and in number of symptomatic drugs change $=50 \%$ & $4 / 36$ & $0 / 14$ \\
Number of responders & $4 / 50$ & $11 \%$ & $0 \%$ \\
Percentage of responders & $8 \%$ & $28 / 36$ & $0 / 14$ \\
Reduction in monthly headache frequency and in number of symptomatic drugs change $>50 \%$ & $0 \%$ \\
Number of responders & $28 / 50$ & $77 \%$ & \\
Percentage of responders & $56 \%$ & \\
\hline
\end{tabular}

Overall, these results confirm the importance of the psychopathological profile in patients with $\mathrm{CM}$ and $\mathrm{MO}$, as the presence of major depression may explain refractoriness to several compounds used in migraine prophylaxis. Managing patients with $\mathrm{CM}$ and $\mathrm{MO}$ with more of one psychiatric comorbidity may be particularly difficult especially when psychiatric disorders are not diagnosed and treated. In particular, our results confirm the findings of previous reports $[10,15]$ suggesting that OCD may be a predictor of poor outcome in these patients, even when drugs effective on depression (like in the present study) are used. Although the presence of OCD is often underestimated in headache patients, this disorder-particularly if not specifically addressed-may favor the compulsive quality of the behaviour, enhancing the drug-seeking tendency due to fear of headache in CM patients, and thus promoting the transformation from episodic migraine to $\mathrm{CM}$, and eventually contributing to the maintenance of chronicity and to the perpetuation of MO.

Appropriate clinical trials are needed to test the efficacy of specific compounds in these patients. However, the results of this open label study may contribute to an adequate insight into this field confirming that assessment of the psychopathological profile should be systematically performed in patients with $\mathrm{CM}$ and $\mathrm{MO}[2,6,7,15]$, and indicating that clinicians should be aware of the positive efficacy of duloxetine in patients with coexisting depression and MO due to $\mathrm{CM}$, as well of the relevant prognostic role of OCD in favoring a poor outcome with persistent disability in these patients.

Conflict of interest I certify that there is no actual or potential conflict of interest in relation to this article.

\section{References}

1. Teixeira AL, Costa EA, da Silva AA Jr, dos Santos IA, Gómez RS, Kummer A, Lauterbach EC (2012) Psychiatric comorbidities of chronic migraine in community and tertiary care clinic samples. J Headache Pain 13(7):551-555
2. Radat F, Swendsen J (2005) Psychiatric comorbidity in migraine: a review. Cephalalgia 25:165-178

3. Buse DC, Silberstein SD, Manack AN, Papapetropoulos S, Lipton RB (2012) Psychiatric comorbidities of episodic and chronic migraine. J Neurol

4. Punay NC, Couch JR (2003) Antidepressants in the treatment of migraine headache. Curr Pain Headache Rep 7(1):51-54

5. D'Amico D, Curone M, Tullo V, Proietti Cecchini A, Mea E, Bussone G (2011) Polytherapy for the prophylaxis of chronic migraine: an Italian survey. Neurol Sci 32(Suppl 1):S185-S188

6. Cupini LM, De Murtas M, Costa C, Mancini M, Eusebi P, Sarchielli P, Calabresi P (2009) Obsessive-compulsive disorder and migraine with medication-overuse headache. Headache 49(7): 1005-1013

7. Curone M, Tullo V, Mea E, Proietti-Cecchini A, Peccarisi C, Bussone G (2011) Psychopathological profile of patients with chronic migraine and medication overuse: study and findings in 50 cases. Neurol Sci 32(suppl 1):S177-S179

8. Vasconcelos LP, Silva MC, Costa EA, da Silva Júnior AA, Gómez RS, Teixeira AL (2008) Obsessive compulsive disorder and migraine: case report, diagnosis and therapeutic approach. J Headache Pain 9(6):397-400

9. Julien D, O'Connor KP, Aardema F (2007) Intrusive thoughts, obsessions, and appraisals in obsessive-compulsive disorder: a critical review. Clin Psychol Rev 27(3):366-383

10. Curone M, D'Amico D, Bussone G (2012) Obsessive-compulsive aspects as predictors of poor response to treatments in patients with chronic migraine and medication overuse. Neurol Sci 33(Suppl 1):S211-S213

11. Headache Classification Committee, Olesen J, Bousser MG, Diener HC, Dodick D, First M, Goadsby PJ, Göbel H, Lainez MJ, Lance JW, Lipton RB, Nappi G, Sakai F, Schoenen J, Silberstein SD, Steiner TJ (2006) New appendix criteria open for a broader concept of chronic migraine. Cephalalgia 26(6):742-746

12. Tfelt-Hansen PC, Hougaard A (2012) Migraine: new US guidelines for preventive treatment of migraine. Nat Rev Neurol $8(8): 419-421$

13. Dhillon S (2013) Duloxetine: a review of its use in the management of major depressive disorder in older adults. Drugs Aging 30(1):59-79

14. Detke MJ, Lu Y, Goldstein DJ, Hayes JR, Demitrack MA (2002) Duloxetine, $60 \mathrm{mg}$ once daily, for major depressive disorder: a randomized double-blind placebo-controlled trial. J Clin Psychiatry 63(4):308-315

15. Luconi R, Bartolini M, Taffi R, Vignini A, Mazzanti L, Provinciali L, Silvestrini M (2007) Prognostic significance of personality profiles in patients with chronic migraine. Headache 47:118-1124 